\title{
Trayectoria histórica de la frontera hispano-mapuche (Chile): la larga duración para la conformación territorial, 1605-1716. Perspectivas desde la geopolítica hispana- parlamentaria
}

\author{
Historical trajectory of the Hispanic-Mapuche border (Chile): the long duration for \\ territorial conformation, 1605-1716. Perspectives from Hispanic-parliamentary \\ geopolitics
}

Cristian González Labra*

\section{RESUMEN}

El artículo hace un estudio de larga duración a la trayectoria histórica de la conformación territorial de la frontera hispano-mapuche entre 1605 y 1803. Se realiza desde la percepción hispana registrada en los parlamentos o encuentros diplomáticos escritos, desde los cuales se observa una transición hacia la integración territorial al sur del Bío-Bío desde inicios del siglo XVIII. La investigación se realiza utilizando la documentación existente generada a partir de la celebración de parlamentos durante la época colonial.

Palabras clave: frontera, fronteras, límite, parlamentos, geopolítica, Bío-Bío.

\section{ABSTRACT}

The article makes a long-term study of the historical trajectory of the territorial conformation of the Hispanic-Mapuche border between 1605 and 1803. It is carried out from the Hispanic perception registered in parliaments or written diplomatic meetings, from which a transition towards territorial integration south of Bío-Bío since the beginning of the 18th century. The research is carried out using the existing documentation generated from the celebration of parliaments during the colonial era.

Keywords: border, borders, limit, parliaments, geopolitics, Bio-Bio.

Recibido: diciembre 2018

Aceptado: noviembre 2019

\footnotetext{
* Magíster en Historia del Tiempo Presente, Universidad Austral de Chile. Email: calacuerda@gmail.com. Se agradece el valioso aporte para la realización de la presente investigación del Doctor Eduardo Téllez Lúgaro.
} 


\section{Introducción}

La investigación intenta demostrar la conformación de la frontera hispano-mapuche en un período de larga duración ${ }^{1}$ (a lo largo del siglo XVII) desde la perspectiva de la geopolítica hispana parlamentaria.

En este sentido, pretende ser un aporte a la comprensión histórica acerca de la conformación territorial de un espacio cuya historicidad produce tensiones en la sociedad de la vieja frontera.

Tras el levantamiento general mapuche que se inició en Curalaba en 1598 se constituyó el límite territorial del río Bío-Bío. Esto conllevó a la creación de una zona de fronteras en el límite natural ${ }^{2}$. La conformación del límite formó parte del plan defensivo hispano bajo la dirección, y primera gobernación de Alonso de Ribera (1601-1605); el que se basó, principalmente, en la construcción de una serie de fuertes a lo largo de dicho límite natural, lo que constituyó dos territorios políticamente diferenciados; al norte, bajo administración estatal hispana; y al sur, de los distintos lof mapuches.

Al estudiar el relato historiográfico fronterizo, desde una perspectiva de larga data, ya en el siglo XVIII Vicente Carvallo y Goyeneche, militar de la frontera e historiador valdiviano, relató acerca de la importancia de mantener al ejército en la línea fortificada del Bío-Bío para terminar así con la guerra ofensiva tras Curalaba, como: "que cesase la guerra ofensiva, sirviéndo de

${ }^{1}$ El término "larga duración" debe entenderse como un período de tiempo aproximado a un siglo, período que permite la consecución de transformaciones a nivel social, político y económico. En contraste, la "corta duración" se refiere a acontecimientos. Ambas categorías, junto a la media y muy larga duración, pueden atribuirse al historiador francés Fernand Braudel. Como texto de referencia: Braudel, Fernand. 2006. "La larga duración", en Revista Académica de Relaciones Internacionales $N^{\circ} 5$, Santiago, pp. 1-36.

2 En este sentido, cabe hacer mención acerca de la distinción histórica de los conceptos: "límite" y "frontera". La historiadora María Ximena Urbina señala que desde la concepción geopolítica hispano-medieval y moderna (época colonial) los límites eran "como lo fueron las marcas entre el mundo cristiano y musulmán" (ver: Urbina C., María. 2009. La Frontera de Arriba en Chile Colonial. Interacción hispano-indígena en el territorio entre Valdivia y Chiloé e imaginario de sus bordes geográficos, 1600-1800, Valparaíso, Ediciones Universidad de Valparaíso, p. 27); es decir, la frontera se entendió como el borde o territorio periférico con respecto al central. Dicho espacio distó de ser una línea, sino más bien, un territorio donde no se había colonizado a la población originaria. En este sentido, de acuerdo a Emir Reitano y Susana Truchelo, es más factible pensar en un límite como división fronteriza durante el siglo XIX durante la época de consolidación de los estados nacionales, los que adoptaron "ópticas estatalistas monolíticas, que se centran en la definición territorial del espacio localizado bajo la autoridad de una única soberanía estatal, a partir de la demarcación de una delimitada y marcada raya de separación" (ver: Reitano, Emir y Truchuelo G., Susana. 2017. "A propósito de Las fronteras en el mundo atlántico (siglos XVI-XIX)", en Reitano, Emir y Truchuelo, Susana (editores). Las fronteras en el Mundo Atlántico (siglos XVI-XIX), Mar del Plata, Universidad Nacional de La Plata, p. 17). De lo anterior, se puede entender que la frontera se constituyó más como un espacio de contacto que a un límite creado por dos entidades políticas. Sin embargo, la frontera hispano-mapuche contó con el Bío-Bío fortificado como un límite divisorio que marcó la separación en sentido norte-sur; ante esto, la existencia de fronteras para los hispanos indicó la existencia de espacios de contacto diferenciados. 
frontera, barrera i línea divisoria las corrientes del Biobio con absoluta prohibicion a los españoles de pasar a la parte meridional del espresado rio"3.

La idea de una línea de frontera no varió con el transcurso del tiempo, ejemplo de esto es lo que estableció Diego Barros Arana en el siglo XIX en su vasta obra "Historia General de Chile", donde señaló que el gobernador Alonso de Ribera a inicios del XVII procuró fortificar dicha línea, para posteriormente irla corriendo al sur". El autor refirió que para 1602 "la tranquilidad parecía restablecida al norte de la línea de frontera planteada por el Gobernador, de tal suerte que los españoles (...) comenzaron a prepararse para trabajar de nuevo sus campos y hasta volver a explotar los lavaderos de oro"5. Dicho avance, finalmente, no se pudo llevar a cabo, por lo que se debieron mejorar las relaciones con los mapuches al norte para asegurar el control del territorio fortificado allende al Bío-Bíó.

Contemporáneo, y en relación a la conformación de una línea de frontera, Sergio Villalobos sostiene que "la derrota y muerte del gobernador Oñez de Loyola en Curalaba (1598) fue seguida por el abandono de todas las ciudades situadas al sur del Biobío (Cañete, Angol, Imperial, Villarrica, Valdivia, Osorno y Santa Cruz)"7, por lo que la colonia tuvo que preparar una línea defensiva en el Bío-Bío por medio de la construcción de una línea de fuertes a cargo de Alonso de Ribera, lo que significó la renuncia del territorio al sur ${ }^{8}$. En este sentido, establece que "Los españoles debieron renunciar a la conquista de la Araucanía y conformarse con el establecimiento de una frontera en el Biobío"9 . Sin embargo, la estabilización de dicha línea la relaciona con los enfrentamientos bélicos hispano-mapuches, por lo que dicha consolidación sucedió "pasada la mitad del siglo XVII, a raíz del tercer gran levantamiento araucano"10, momento en que las relaciones socioeconómicas reemplazaron a las bélicas ${ }^{11}$.

José Bengoa es otro que se ha referido a la consolidación de una línea defensiva, presentando a Ribera como co-protagonista. En este sentido, "Ribera percibió durante su primer mandato que se estaba consolidando una frontera y colaboró en ello. Construyó fuertes en el Bío Bío y prepraró un ejército que protegiera ese límite"12.

\footnotetext{
${ }^{3}$ Carvallo G, Vicente. 1875. Descripción históricio-geográfica del Reino de Chile, Santiago, Imprenta del Ferrocarril, p. 252.

${ }^{4}$ Barros A., Diego. 1999. Historia General de Chile, Tomo III, Santiago, Editorial Universitaria, p. 278.

5 Ibíd, p. 280.

${ }^{6}$ Ibíd, p. 290.

${ }^{7}$ Villalobos R., Sergio. 2003. Chile y su historia, Santiago, Editorial Universitaria, p. 91.

8 ídem.

9 Ídem.

${ }^{10}$ Villalobos R., Sergio. 1995. Vida fronteriza en Chile: el mito de la Guerra de Arauco, Santiago, Andrés Bello, p. 255.

11 Ibíd, p. 249.

12 Bengoa A., José. 2003. Historia de los antiguos mapuches del sur, Santiago, Catalonia, p. 343.
} 
Andrea Ruiz-Esquide también se ha referido a la línea de frontera asimilándola al Bío-Bío, planteando que se conformó a comienzos del siglo XVII producto "de la rebelión indigena, que en 1598 habia destruido las siete ciudades del sur y había significado la muerte del gobernador Martín García Oñez de Loyola"13. Ante esto, los hispanocriollos habrían optado por consolidar el territorio al norte de dicha línea ${ }^{14}$.

María Angélica Illanes, con relación a la consolidación de un nuevo espacio fronterizo, señala: "el espacio fronterizo (...) quedó delimitado en el siglo XVII por el Biobío al sur"15. Illanes establece que la creación de dicho espacio (por causa del levantamiento general en Curalaba) dio fin al expansivo siglo XVI español en Chile, "debiendo replegar los hispanos sus fuerzas del Biobío al norte y de Chiloé al sur"16. Como respuesta, refiere que se creó un ejército permanente y se aprobó la esclavitud indígena en 1608, firmándose en 1641 las Paces de Quillín, con lo que se "reconoció la libertad de los mapuche de Chile/Sur"17.

Fernando Venegas realiza una interesante apreciación respecto a la frontera en el Bío-Bío tras Curalaba. Se refiere a que aquéllo solo ocurrió en un estado inicial, pues en un período posterior, por la disposición de los fuertes en posición estratégica, se erigiría en efecto a lo largo del Bío-Bío y en territorios situados en la Araucanía (fuertes de San Ignacio de Boroa y Paicaví en 1606, Encarnación en 1666 y Repocura en 1894) ${ }^{18}$.

De la revisión historiográfica es posible observar el límite territorial del Bío-Bío como el eje constitutivo del espacio fronterizo. Sin embargo, la documentación parlamentaria hispanomapuche, la que exhibe la perspectiva hispana desde los encuentros diplomáticos escritos, muestra la existencia tanto de una frontera como de múltiples fronteras al sur del Bío-Bío. En este sentido, la documentación hace referencia a una multiplicidad de espacios fronterizos, especialmente durante la primera mitad del siglo XVII, mientras que durante la segunda mitad se iría conformando un espacio fronterizo uniforme, para consolidar dicha conformación en el XVIII.

Al tenor de lo expuesto, en la presente investigación se realiza un estudio en profundidad a los parlamentos hispano-mapuches, indagando acerca de la percepción que tuvieron los hispanos acerca del espacio de fronteras en el Bío-Bío.

\footnotetext{
${ }^{13}$ Ruiz E., Andrea. 1993. Los indios amigos en la frontera araucana, Santiago, DIBAM, p. 12.

14 Ídem.

15 Illanes O., María. 2014. "La cuarta frontera valdiviana. El caso del territorio valdiviano (Chile, XVII-XIX), en Atenea N 509, Concepción, p. 228.

${ }^{16}$ Ibíd, p. 233.

17 Ídem.

18 Venegas E., Fernando. 2014. De Tralca-Mawida a Santa Juana. Despliegue Histórico de una Localidad en la Frontera del Biobío, Valparaíso, Ediciones Universitarias de Valparaíso, p. 37.
} 
La investigación se realiza estudiando el registro completo existente a lo largo del período colonial posterior a Curalaba, entre 1605 y 1803, el que consta de 37 tratados manuscritos; esto, con el fin de tener una visión de largo plazo desde la percepción hispana acerca de la existencia de una frontera múltiple y, posteriormente, uniforme al sur del Bío-Bío. Desde le perspectiva del método; primero, se analizan los parlamentos; para luego, estudiar la trayectoria histórica desde la geopolítica hispana. En este sentido, los parlamentos se utilizan ya que son una rica fuente desde la cual entender la geopolítica. Por último, cabe hacer mención acerca del texto recopilatorio de los encuentros formales: "Los Parlamentos HispanoMapuches, 1593-1803: Textos Fundamentales"19 ${ }^{19}$ en el que se recopilan cronológicamente los registros de los encuentros; además, se recurre a literatura histórica afín ${ }^{20}$.

19 Zavala. 2015. Los Parlamentos Hispano-Mapuches, 1593-1803: Textos Fundamentales, Temuco, Ediciones Universidad Católica de Temuco.

${ }^{20}$ Como antecedentes de estudios en temática fronteriza, ver: Alonso A., Icíar y Payás P., Gertrudis. 2009. "La mediación lingüística institucionalizada en las fronteras Hispano-mapuche e Hispano-árabe: ¿un patrón similar?", en Historia N 42, Santiago, pp. 185-201; Barros A., Diego. 1999. Historia General de Chile, Tomo III, Santiago, Editorial Universitaria; Barros A., Diego. 1999. Historia General de Chile, Tomo IV, Santiago, Editorial Universitaria; Bengoa A., José. 2000. Historia del pueblo mapuche. Siglos XIX y XX, Santiago, LOM; Bengoa. 2003. Historia de los...; Bengoa C., José. 2007. El tratado de Quilín, Santiago, Catalonia; Carreño S., Luis. 2009. "Relaciones fronterizas y violencia en la plaza fuerte de Valdivia. Siglo XVIII", en Espacio Regional Nº 6, Osorno, pp. 13-22; Cerda H., Patricia. 1997. Fronteras del Sur. La Región del Bío-Bío y la Araucanía chilena, 1604-1883, Temuco, Ediciones Universidad de la Frontera; Curivil P., Ramón; Payás, Gertrudis; y Zavala, José. 2014. "La palabra "Parlamento" y su equivalente en mapudungún en los ámbitos colonial y republicano. Un estudio sobre fuentes chilenas bilingües y de traducción", en Historia $\mathrm{N}^{\circ} 47$, Santiago, pp. 355-373; Dillehay, Tom y Zavala, José. 2010. "El "Estado de Arauco" frente a la conquista española: estructuración sociopolítica y ritual de los araucano-mapuches en los valles nahuelbutanos durante los siglos XVI y XVII", en Chungará N² 2, Arica, pp. 433-450; Dillehay, Tom; Payás P., Gertrudis; y Zavala C., José. (2013). "EI requerimiento de Martín García Óñez de Loyola a los indios de Quilacoya, Rere, Taruchina y Maquegua de 1593, testimonio oficial de Parlamentos Hispano-mapuches tempranos", en Memoria Americana N²1, Buenos Aires, pp. 235-268; Dillehay, Tom; Le Bonniec, Fabien; Payás P., Gertrudis; y Zavala C., José (editores). 2015. Los parlamentos hispano-mapuches como espacios de reconocimiento del Otro enemigo: huellas históricas, lingüísticas y territoriales, Salamanca, Ediciones Universidad de Salamanca, pp. 35-48; Foerster G., Rolf y Vergara M., Jorge. (1993). "¿Relaciones interétnicas o relaciones fronterizas?", en Revista de Historia Indígena N 1, Santiago, pp. 9-33; Góngora. 1966. Vagabundaje y sociedad...; Illanes. 2014. La cuarta frontera...; Leiva. 1985. El primer avance...; León S., Leonardo. 1990. Maloqueros y Conchavadores en Araucanía y las Pampas, 1700-1800, Temuco, Ediciones Universidad de la Frontera; León S., Leonardo. 1993. El Parlamento de Tapihue, 1774, Santiago, Ediciones Rehue; Méndez B., Luz. 2014. "Diálogo entre españoles e indígenas en los Parlamentos de los siglos XVI y XVII", en Cornejo, Alejandra (editores). Coyunturas pasadas y presentes de los pueblos originarios, Santiago, Catalonia, pp. 13-81; Payás P., Gertrudis y Zavala C., José. (2015). "Ambrosio O'Higgins y los Parlamentos Hispano-mapuches, 1771-1803: política indígena, escritura administrativa y mediación lingüístico-cultural en la época borbónica chilena", en Memoria Americana $\mathrm{N}^{\circ}$ 23, Buenos Aires, pp. 103-136; Pinto R., Jorge. 1988. "Frontera, misiones y misioneros en Chile. La Araucanía, 1600-1900", en Casanova, Holdenis; Matthei, Mauro; Pinto, Jorge; y Uribe, Sergio. Misioneros en la Araucanía, Temuco, Ediciones Universidad de la Frontera; Pinto R., Jorge. 1996. Araucanía y Pampas. Un mundo fronterizo en América del Sur, Temuco, Ediciones Universidad de la Frontera; Ruiz. 1993. Los indios amigos...; Urbina. 2009. La Frontera de...; Villalobos. 2003. Chile y su...; Zavala C., José. 2005. "Aproximación antropológica a los parlamentos hispano-mapuches del siglo XVIII", en Revista Austerra Nํ2, Santiago, pp. 49-58. 


\section{Parlamentos hispano-mapuches}

Los parlamentos conllevaron interrelaciones a ambos lados del límite divisorio del Bío-Bío, involucrando, principalmente, a quienes detentaban el poder político y militar. Leonardo León entiende que los parlamentos fueron los instrumentos formales de comunicación que permitieron lograr acuerdos generales, los que involucraron a la mayor parte de la sociedad regional; esto, ya que estvieron presentes los principales líderes de ambas sociedades, los que, además, establecieron las reglas para el intercambio fronterizo ${ }^{21}$. Es decir, que estos encuentros conllevaron a la consecución de acuerdos en búsqueda de la estabilización del territorio regional, caracterizado por tener un alto grado de interrelaciones socioeconómicas. Dichos acuerdos permiten entender los distintos mecanismos que se utilizaron para que existiesen, aunque fuese temporalmente, grados de estabilidad, al tener la firme creencia que el territorio del enemigo era el propio.

Desde la perspectiva de su trayectoria histórica, León señala que el parlamento apareció a fines del siglo XVI, madurado durante el XVII, formalizado durante el XVIII y alcanzado su máxima convocatoria en el último cuarto del XVIII e inicios del XIX ${ }^{22}$. Del escrito se desprende que la conformación del parlamento tomó casi todo el período colonial en Chile. Pero esta instancia de relaciones regionales, ¿qué características tenía? Con respecto a esto, José Zavala realiza una caracterización, la que permite entender de una manera sintética su complejidad, al establecer que eran convenios entre partes, el ser un espacio culturalmente negociado y que se constituía como un lugar de comunicación inter-lingüística ${ }^{23}$, de lo que se deduce que existían relaciones simétricas. Sin embargo, hay que tener presente que el parlamento del que se hace mención y estudio es el escrito; en este sentido, la sociedad mapuche no hizo uso del documento escrito, por lo que su cultura ágrafa no nos permite acceder a su manera de relacionarse previa al parlamento general en estudio. El mismo autor citado, junto a Gertrudis Payás y Ramón Curivil, dan cuenta del coyag, el que tuvo una forma discursiva para relacionarse intraétnicamente en la sociedad mapuche ${ }^{24}$, siendo el uso de la palabra el instrumento de mediación por excelencia empleado por los loncos de las parcialidades participantes en las juntas generales.

\footnotetext{
${ }^{21}$ León S., Leonardo. 1993. El Parlamento de Tapihue, 1774, Santiago, Ediciones Rehue, p. 7. 22 Ibíd., p. 8.

23 Payás P., Gertrudis y Zavala C., José. (2015). "Ambrosio O’Higgins y los Parlamentos Hispano-mapuches, 17711803: política indígena, escritura administrativa y mediación lingüístico-cultural en la época borbónica chilena", en Memoria Americana $\mathrm{N}^{\circ} 23$, Buenos Aires, p. 17.

24 Curivil P., Ramón; Payás, Gertrudis; y Zavala, José. 2014. "La palabra "Parlamento" y su equivalente en mapudungún en los ámbitos colonial y republicano. Un estudio sobre fuentes chilenas bilingües y de traducción", en Historia $N^{\circ} 47$, Santiago.
} 
Por otro lado, el establecimiento del parlamento como mecanismo formal-regional de mediación fronteriza se concretó durante el siglo XVII. Este mecanismo tuvo su origen, de acuerdo a Luz Beltrán, en la ciudad de Concepción el 22 de noviembre de 1593, entre el gobernador Martín García Óñez de Loyola y los mapuches próximos a esta ${ }^{25}$. Cabe agregar el intento jesuita del Padre Luis de Valdivia de forjar relaciones con las Reguas de Catiray durante la Guerra Defensiva, momento en que se recurrió a las comunicaciones interétnicas como mecanismo pacífico de relación en la zona. En este sentido, es importante destacar que el Estado Monárquico español dio un reconocimiento oficial poco tiempo después con la celebración del Parlamento de Quillín en 1641 durante la administración del gobernador Francisco López de Zúñiga y Meneses (Marqués de Baydes). Bengoa es uno de los que ha brindado mayor peso histórico a este encuentro, al establecer que lo sucedido tuvo una importante repercusión para la historia de la sociedad chilena por la división fronteriza en el Bío-Bío ${ }^{26}$. Lo cierto es que después de Quillín se llevaron a cabo numerosos parlamentos en lo que restó del período colonial, los que fueron conformando un mecanismo oficial de comunicación fronteriza en cuanto a la realización de las juntas (protocolos, agasajos, pagos de sueldos, etc.) y el registro escrito ${ }^{27}$.

\section{Trayectoria fronteriza desde los parlamentos hispano-mapuches, 1605-1803}

Como se mencionó, el primer parlamento se celebró en 1593, lo que exhibe el lato empleo de este mecanismo para aliviar las relaciones interétnicas en el tenso escenario bélico. Para 1605 fueron siete los llevados a cabo. Se celebraron en Concepción, Yumbel y los Fuertes de Santa Inés de Monterrey, Santa Margarita de Austria, San llifonso de Arauco, Santa Fe de la Paz y Buena Esperanza. Cobró especial relevancia el llevado a cabo en Concepción, ya que fue el primero en que se dio cuenta de la idea de la existencia de las fronteras o la frontera. En este

\footnotetext{
${ }^{25}$ Méndez B., Luz. 2014. "Diálogo entre españoles e indígenas en los Parlamentos de los siglos XVI y XVII", en Cornejo, Alejandra (editores). Coyunturas pasadas y presentes de los pueblos originarios, Santiago, Catalonia, p. 16.

${ }^{26}$ Bengoa C., José. 2007. El tratado de Quilín, Santiago, Catalonia, p. 7.

${ }^{27}$ En este sentido, cabe señalar la segmentación que hacen Dillehay, Le Bonniec, Payás y Zavala de la trayectoria parlamentaria, la cual permite ver un desarrollo de los encuentros formales. Dicha clasificación contempla tres etapas; una temprana, en el último decenio del siglo XVI; otra de consolidación, desde el cuarto decenio hasta el último del XVII; y una clásica, durante el XVIII. Con respecto a esto, establecen: "Es posible distinguir tres fases en el desarrollo de los parlamentos: En primer lugar, una etapa temprana (último decenio del siglo XVI), en la cual el parlamento se desarrolla en un ámbito local, es decir la concertación se realiza entre españoles y grupos indígenas geográficamente acotados a un acotados aun valle o a valles interconectados. Posteriormente, una etapa de consolidación (siglo XVII, desde el cuarto decenio hasta el último). Durante esta época el parlamento se consolida como el principal mecanismo de negociación política entre españoles y conglomerados indígenas d eamplios terirtorios de la Araucanía y delo svalles andinos próximos. Finalmente, una etapa clásica (siglo XVIII). En esta fase el parlamento apatrece bien institucionalizado y formalizado, con una dinámica de realización bien rodada e integrada a la institucionalidad fronteriza española". Ver: Dillehay, Tom; Le Bonniec, Fabien; Payás P., Gertrudis; y Zavala C., José (editores). 2015. Los parlamentos hispano-mapuches como espacios de reconocimiento del Otro enemigo: huellas históricas, lingüísticas y territoriales, Salamanca, Ediciones Universidad de Salamanca, p. 35.
} 
sentido, se estableció: "mando el dicho governador a todos los corregidores y capitanes de guerra y de frontera diesen alguna noticia á todos los caciques (...) que an estado revelados de quel dicho Governador les quería hablar en todas las dichas fronteras" ${ }^{\prime 28}$. Si bien las referencias son ambiguas, permiten entrever la idea de una barrera física entre ambas sociedades.

De acuerdo a lo que se indicó, desde la historiografía se ha entendido que el principal ideólogo de la preparación de una línea de defensa basada en el límite natural del Bío-Bío y la construcción de una serie de fuertes a lo largo de este fue el gobernador Alonso de Ribera, quien dispuso, además, que los campos hispanos próximos (vecinos de Concepción y Chillán) debían proveer del alimento necesario para la subsistencia de las guarniciones, aspecto que en el futuro demostró no ser así, ya que se tuvo que recurrir constantemente a los intercambios con las poblaciones mapuches al sur. Lo anterior se debe contextualizar con la emergencia por frenar el avance de los mapuches liderados, entre otros, por los toquis Pelantaro, Huaiquimilla y Anganamón.

Entre 1612 y 1617 los acuerdos formaron parte de la mencionada Guerra Defensiva, la que tuvo al padre jesuita Luis de Valdivia como uno de sus protagonistas. El esfuerzo jesuita buscó por medio de la evangelización concretar la paz, esfuerzo que finalmente no perduró. Desde la perspectiva parlamentaria, uno de los encuentros más importantes fue el que se llevó a cabo en Catiray en 1612 (celebrado en las "Reguas de Catiray"), el que fue fundamental para el establecimiento del mecanismo formal para las relaciones fronterizas. Allí, Valdivia se dirigió a las alturas de la Cordillera de Nahuelbuta, lugar-fuerte de los jefes guerreros mapuches en el "Estado de Arauco"29. En este lugar se afirmó la importancia como barrera física del Bío-Bío junto a la construcción de la línea de fuertes a lo largo de este. En este sentido, se señaló: "que es El Señor Alonso de rivera aquel a quien por ser tan grande Capitan L[I]amavades La peña Fuerte el qual iba ganando la tierra sin bolber un pie atras el que dejo ganado todo biobio, y tan fortificado"30. Esta referencia al Bío-Bío se repitió en los siguientes parlamentos de 1612, los que tuvieron lugar en los fuertes de San Ilifonso de Arauco y Paicaví, al referirse a la barrera natural y física como "la raya"31.

\footnotetext{
${ }^{28}$ Parlamento de Concepción de 1605, AGI, Patronato, 228, fs. 1-31; APUD Zavala. 2015. Los Parlamentos HispanoMapuches..., p. 47.

${ }^{29}$ El parlamento se refirió al lugar como Catiray. De acuerdo a Dillehay y Zavala, Mareguano-Catiray, Arauco, Tucapel y Purén-Lumaco son cuatro grandes cuencas que se desprenden de la Cordillera de Nahuelbuta y que, desde la perspectiva hispana, fue visto como el "Estado de Arauco" debido a la existencia de grandes alianzas. Ver: Dillehay, Tom y Zavala, José. 2010. "El "Estado de Arauco" frente a la conquista española: estructuración sociopolítica y ritual de los araucano-mapuches en los valles nahuelbutanos durante los siglos XVI y XVII", en Chungará №2, Arica, pp. 436-447.

${ }^{30}$ Parlamento de Catiray de 1612, ANHCh, FJ, vol. 93, fs. 61-64; APUD Zavala. 2015. Los Parlamentos HispanoMapuches..., p. 73.

${ }^{31}$ Parlamento de San Ilifonso de Arauco de 1612, ANHCh, FMV, vol. 2, fs. 20-24; APUD Zavala. 2015. Los Parlamentos Hispano-Mapuches..., p. 80.
} 
Para 1617, en Nacimiento, el carácter múltiple de las fronteras se hizo evidente. Fueron varias las referencias acerca de la multiplicidad de dichos espacios en "la raya" del Bío-Bío. A modo de ejemplo, vale mencionar el señalamiento hispano del espacio sureño, el que es representativo de otros casos; "los enseñase y baptizase y los q[ue] de aquí adelante naciesen. Mostráronse agradecidos los caciques que embié a llamar de todas las fronteras de Biobío p[ar]a este parlam[en]to"32. En contraste, se señaló la idea de la frontera, aunque en menor proporción y de manera ambigua. En este sentido, dichas referencias, las que hablan en el sentido uniforme, no hacen un señalamiento explícito a una vinculación macrorregional, sino más bien, a lugares específicos. Por ejemplo, es posible señalar las expresiones: "Porque no entrasen acá dentro tantos mensajeros a buscarme, porq[ue] podían ser espías con color de paz, lo qual se euitaba hallándome en la frontera"33 e "y que las proui[nci]as de arriba respondieron q[ue] ellas estauan quietas y nunca se ynquietauan, si no era qu[an]do la proui[nci]a de Purén, q[ue] estaua en frontera de guerra, les pedia gente p[ar]a hazerla" ${ }^{34}$. El parlamento citado, llevado a cabo en Nacimiento, es interesante, ya que rompe con la visión tradicional del espacio fronterizo al sur del Bío-Bío como una frontera uniforme. Al contrario, las múltiples referencias exhiben, desde la perspectiva hispana, su visión acerca de la multiplicidad de pueblos al sur.

Un poco más de dos décadas después, en 1641 y 1647, tuvieron lugar dos parlamentos, ambos llevados a cabo en el Valle de Quillín, los que han tenido un lugar central para la historiografía ${ }^{35}$. El primero de ellos ha sido sin duda el que ha cobrado mayor relavancia, el que se llevó a cabo con la concurrencia del Gobernador de la Capitanía General, Francisco López de Zúñiga y Meneses, Marqués de Baydes, quien es descrito como uno de los principales responsables para su realización. Desde allí se inició una formalización de las relaciones parlamentarias, las que continuaron hasta 1803 en Negrete. De los principales aspectos a destacar está la valoración que se hizo desde el Estado Monárquico a los habitantes allende el Bío-Bío, reconociendo su condición de pueblo libre a cambio de que fueran vasallos del rey. Por supuesto que estas estipulaciones buscaron crear una soberanía (ficticia) de la Corona al sur de dicho límite, lo que significó que dicho territorio le pertenecería de manera nominal, por lo que el control en la práctica lo tendrían los distinos lof. Quillín, vale mencionar, se encuentra ubicado en medio de la actual Araucanía, en lo que es la Provincia de Cautín, este dato

\footnotetext{
32 Parlamento de Nacimiento de 1617, Díaz Blanco, 2011, 420-435; APUD Zavala. 2015. Los Parlamentos HispanoMapuches..., p. 102.

33 Parlamento de Nacimiento de 1617, Díaz Blanco, 2011, 420-435; APUD Zavala. 2015. Los Parlamentos HispanoMapuches..., p. 97.

34 Parlamento de Nacimiento de 1617, Díaz Blanco, 2011, 420-435; APUD Zavala. 2015. Los Parlamentos HispanoMapuches..., p. 99.

${ }^{35}$ Como ejemplo a destacar, ver: Bengoa C., José. 2007. El tratado de Quilín, Santiago, Catalonia.
} 
geográfico es interesante si se contrasta con la documentación existente. En este sentido, del texto del parlamento de 1641 se puede leer: "habiéndolas oido el Marqués, les representó las que habia de conveniencia para que no fuese otro el lugar (...) sino el ya señalado de Quillin, por ser en medio de las fronteras y sitio neutral y despoblado"36. Esto es relevante, ya que la descripción que se hace del lugar al estar situado "en medio de las fronteras", permite visualizar que la idea de la frontera no se limitaba a la zona aledaña al Bío-Bío, sino que al territorio bajo control mapuche. Así también, en el lenguaje del parlamento siguiente, de 1647, se continuó plasmando la idea de una multiplicidad de sectores fronterizos, como: "de parte de algunos toquis generales, y cassiques prinsipales de las fronteras de la guerra" ${ }^{137} \mathrm{O}$ "de las demaz [toquis, lonkos e indios amigos] de nuestras fronteras" 38 .

Para la década siguiente se produjo un importante levantamiento mapuche por causa, de manera principal, de la esclavización de los "indios en guerra" durante el gobierno de Acuña y Cabrera. Como resultado, la ofensiva mapuche llegó hasta el Maule, lo que puso en riesgo la existencia de la misma colonia. En 1662 se reanudaron los encuentros formales en el Parlamento de Santa María de Guadalupe, en el que se estableció, con relación a la recuperación de los territorios perdidos al sur, que: "los dichos señores y discurrido largamente en la matería con el celo y atençion que pedia dijeron todos uniformemente que ynportava al servicio de su Magestad y recuperacion de nuestras fronteras reçivir los dichos caciques y demas" ${ }^{139}$. También se refirieron a la prohibición de borracheras y juegos, es decir, a la sociabilidad mapuche en las fronteras. En este sentido: "Que no puedan juntarse a publicas borracheras ni a juegos de chueca sin lizencia del Señor governador o cavo general que governare Las fronteras o de sus capitanes porque asi conbiene al serviçio de su Magestad"40. En contraste, se hizo referencia al territorio fronterizo para señalar la "frontera del estado de arauco"41, relacionado con el tercio de Santa María de Guadalupe y Venavides, los que debían pedir un permiso (españoles), o licencia, "al maestro de campo Governador o cavo de la frontera por la parte Donde binieren para poder haçer su biaje Diçiendo y explicando primero

\footnotetext{
${ }^{36}$ Parlamento de Quillín de 1641 - Impreso de Madrid, Francisco Maroto, 1642 - (Reedición de 1879: pp.235-278) APUD Zavala. 2015. Los Parlamentos Hispano-Mapuches..., p. 113.

${ }^{37}$ Parlamento de Quillín de 1647 - AGI, Chile, 21, fs. 1-16v; APUD Zavala. 2015. Los Parlamentos Hispano-Mapuches..., p. 123.

${ }^{38}$ Parlamento de Quillín de 1647 - AGI, Chile, 21, fs. 1-16v; APUD Zavala. 2015. Los Parlamentos Hispano-Mapuches..., p. 127.

39 Parlamento de Santa María de Guadalupe de 1662 - AGI, Chile, 22, fs. 1-6; APUD Zavala. 2015. Los Parlamentos Hispano-Mapuches..., p. 137.

40 Parlamento de Santa María de Guadalupe de 1662 - AGI, Chile, 22, fs. 1-6; APUD Zavala. 2015. Los Parlamentos Hispano-Mapuches..., p. 138.

${ }^{41}$ Parlamento de Santa María de Guadalupe de 1662 - AGI, Chile, 22, fs. 1-6; APUD Zavala. 2015. Los Parlamentos Hispano-Mapuches..., p. 137.
} 
a donde y como ban"42. Lo anterior fue común en la zona, donde el paso por los fuertes se impuso como una obligación en las disposiciones parlamentarias, como una medida de control a la sociedad fronteriza en ciernes.

En la década siguiente, en el Parlamento de Malloco de 1671, se hizo alusión a la evangelización que se debía hacer en las fronteras y, en relación a esto, refiriéndose a los "enemigos": "Les enseñen la doctrina y Reçivan el Santo Sacramento (...) que tan faltos estan del y muchos de los que viven entre nosotros Lo estan por no haver quien Los administre en las fronteras Vecinas a los enemigos"43. En este caso, se consideró la doctrina cristiana como factor para un clima de estabilidad en la zona, mostrando la constante intención española por colonizar a los "herejes" por una vía alternativa a la bélica.

A fines del siglo XVII, y recurriendo a lo que establece Villalobos, comenzó una etapa caracterizada por el desarrollo de las relaciones económicas, dejando de ser la guerra la característica principal. La guerra habría durado hasta los primeros años de la década de 1680 , siendo la causa principal el abandono gradual de las hostilidades por el creciente proceso de acercamiento y contactos múltiples que superaron a los encuentros armados ${ }^{44}$. De esta forma, a pesar del carácter bélico del territorio durante buena parte del siglo, las relaciones socioeconómicas fueron imponiéndose de manera progresiva como alternativa a una confrontación que no encontraba un vencedor. En los parlamentos celebrados a fines de siglo (Yumbel, 1692; Concepción, 1693; Choque-Choque, 1694; y Purén, 1698), es posible señalar, como aspectos a destacar con relación a la temática fronteriza, las referencias en materia defensiva en los fuertes y la intervención de los Capitanes de Amigos en el territorio mapuche como agentes de control y justicia. Con relación a esto, es pertinente ejemplificar con tres juntas generales; durante el Parlamento de Yumbel de 1692, se estableció: "no aian de quitarle la vida ni quemarles sus casas como acostumbran sino dar parte a su capitan o ministro prinçipal de la frontera para que se les haga justiçia"45; en el Parlamento de Choque-Choque en 1694: "les avia puesto Su señoria Capitanes que los governasen en sus reduciones y les administrasen Justicia y asi mesmo los Cavos y ministros de las plazas de la Frontera los avian oido y echo Justicia"46 o "Y el sargento maior Bartholome Perez de Villagra cavo de la Plaza y fronteras de

\footnotetext{
42 Parlamento de Santa María de Guadalupe de 1662 - AGI, Chile, 22, fs. 1-6; APUD Zavala. 2015. Los Parlamentos Hispano-Mapuches..., p. 139.

43 Parlamento de Malloco de 1671, AGI, Chile, 56, fs. 1-1v; APUD Zavala. 2015. Los Parlamentos Hispano-Mapuches..., p. 165.

${ }^{44}$ Villalobos. 2003. Chile y su...

45 Parlamento de Yumbel, de 1692, BNCh, Ms.M, t. 315, fs. 1-55; APUD Zavala. 2015. Los Parlamentos HispanoMapuches..., p. 174.

${ }^{46}$ Parlamento de Choque Choque de 1694, AGI, Chile, 105, fs. 1-11; APUD Zavala. 2015. Los Parlamentos HispanoMapuches..., p. 196.
} 
Puren: y otros muchos Cavos y Capitanes actuales y reformados"47; y en el Parlamento de Purén de 1698: "que a un mismo tiempo â tenido especial Cuidado en poner en las fronteras españoles experimentados en el modo de Governarlos quitandolos de sus Conveniencias solo a fin de que los mantengan en paz y en justicia"48. Los parlamentos antes expuestos exhiben la existencia de una frontera o de múltiples fronteras. En este sentido, el parlamento celebrado en ChoqueChoque es quizás el más interesa interesante, ya que se refirió a las "fronteras de Puren", denotando la pluralidad de territorios en un área al sur del Bío-Bío y, además, local.

A inicios del siglo XVIII cambió la lógica parlamentaria con la llegada de los Borbones al poder monárquico hispano. Con estos se buscó un mayor control territorial, lo que se tradujo, para el caso de la colonia chilena, en aprovechar las instancias parlamentarias para mantener una convivencia fronteriza en un territorio históricamente inestable desde mediados del XVI. En la carta de 1716 el gobernador de Chile, Juan Andrés de Ustáriz, al rey hispano, Felipe V, informó acerca de la celebración del Parlamento de Tapihue llevado a cabo ése mismo año. En la misiva destacó el comentario acerca de la característica de frontera para las zonas próximas al Bío-Bío, la que estaría compuesta por Purén, Tucapel, Arauco y Yumbel. Se relató: "y de ordenes á los Cabos de Puren, Tucapel, Arauco, y Yumbel para que hiciesen concurrir á los Caciques Toquis y demás principales de la frontera al dicho sitio"49.

Para la década siguiente se rompió el breve período de paz en la zona, al tener lugar un importante levantamiento mapuche debido, en buena medida, a los abusos de los hispanos, destacando el robo de hijos. En parte, el Parlamento de Negrete celebrado en 1726 permitió pactar y reducir la tensión en la zona por medio del abandono de los fuertes al sur del Bío-Bío. En el parlmento se estableció la destrucción de los fuertes al sur del límite natural y su reinstalación al norte (del mencionado límite), estipulándose que se llevaría a cabo bajo el arbitrio del gobernador Gabriel Cano de Aponte. Se estipuló: "el mismo paraje o en otros mas a propocito para la combeniencia y amparo de los mismos yndios" 50 , además de que estarían "obligados los yndios en cuya Jurisdicion se pusiera a concurrir al travajo y los caciques ha acerlos asistir a la redificacion y los españoles a Mantenerlos con el mantenimiento acostumbrado quando se piden mitas" ${ }^{51}$. Dos décadas más tarde el mecanismo parlamentario

\footnotetext{
47 Parlamento de choque choque de 1694, AGI, Chile, 105, fs. 1-11; APUD Zavala. 2015. Los Parlamentos HispanoMapuches..., p. 195.

48 Parlamento Purén de 1698, AGI, Chile, 129, fs. 1-11v; APUD Zavala. 2015. Los Parlamentos Hispano-Mapuches..., p. 208.

49 Parlamento de Tapihue de 1716, BNCh, Ms.M, t. 178, fs. 105-109; APUD Zavala. 2015. Los Parlamentos HispanoMapuches..., p. 215.

${ }^{50}$ Parlamento de Negrete de 1726, ANHCh, FV, vol. 251, fs. 174-193; APUD Zavala. 2015. Los Parlamentos HispanoMapuches..., p. 221.

${ }^{51}$ Parlamento de Negrete de 1726, ANHCh, FV, vol. 251, fs. 174-193; APUD Zavala. 2015. Los Parlamentos HispanoMapuches..., p. 221.
} 
mostró ser la herramienta más idónea para mantener la paz, la cual había sido esquiva desde hacía más de un siglo. Muestra de esto es lo que se parlamentó en 1746, en Tapihue, donde el gobernador Domingo Ortiz de Rozas manifestó la importancia de mantener buenas relaciones, además de señalar a la zona como de frontera. A este respecto: "Uno de los principales fines que me conduxeron a la frontera, fue el de hazer Parlamento General a los Yndios para establezerles en la paz y buena correspondencia que deben observar con los españoles"52. Con esto, por lo menos si se atiene a lo que se estableció, Ortiz continuó con la política "pacifista" de Cano de Aponte de mantener buenas relaciones tras el fuerte levantamiento de 1723, lo que sería una práctica regular en lo que restó del período colonial, es decir, el fortalecimiento del mecanismo parlamentario.

A inicios de la la segunda mitad de siglo, en el parlamento celebrado en Concepción en 1759, destacó la forma en que se relacionó al territorio de frontera como un territorio ultra Bío-Bío, al señalarse: "En el Parlamento General, que siguiendo el antiguo rito de este Govierno, hice à los Yndios à mi ingreso à servirlo; algunos de los Caziques mas retirados de la Frontera"53. En este caso se puede percatar la existencia de un territorio de mayor extensión, esto, por la lejanía desde la línea del Bío-Bío. Si se "esfuerza" la observación parlamentaria, incluso se podría pensar que se refieren a un espacio que podría abarcar hasta el río Toltén o incluso a cercanías del pueblo-presidio de Valdivia. Por supuesto, un aspecto que no pudo soslayarse fue la mantención de la línea de fuertes, haciéndose referencia que: "observando el desbelo con que desde el primer dia sin intermision travajo en reforzar la Frontera, cuios Fuertes y Plazas (...) se hallan en el estado mas respectable que con admiracion de todos"54, exhibiendo, por un lado, la fuerza militar como mecanismo para asegurar el control y, por otro, que el mecanismo parlamentario no aseguraba por sí solo la paz. En este sentido, en el Parlamento de Santiago de 1760 se continuó con la idea de la defensa fortificada de dicho espacio ${ }^{55}$.

Siete años más tarde ocurrió el último enfrentamiento hispano-mapuche de importancia en la frontera, el que sucedió entre 1766 y 1774 . El conflicto hizo que la Corona intensificara su política parlamentaria como mecanismo de convivencia fronteriza, por lo que los gobernadores siguientes hicieron importantes esfuerzos, especialmente en el plano económico, los que tuvieron relación, por ejemplo, con los preparativosde los parlamentos y los agasajos a los

\footnotetext{
52 Parlamento de Tapihue de 1746, BNCh, MBA, t. 37, fs. 752-779; APUD Zavala. 2015. Los Parlamentos HispanoMapuches..., p. 247.

53 Parlamento de Concepción de 1759, BNCh, MBA, t. 37, fs. 792-805; APUD Zavala. 2015. Los Parlamentos HispanoMapuches..., p. 257.

${ }^{54}$ Parlamento de Concepción de 1759, BNCh, MBA, t. 37, fs. 792-805; APUD Zavala. 2015. Los Parlamentos HispanoMapuches..., p. 257.

55 Parlamento de Santiago de 1760, BNCh, MBA, t. 37, fs. 1000-1035; APUD Zavala. 2015. Los Parlamentos HispanoMapuches..., p. 263.
} 
principales loncos. De entre los gobernadores del período destacó como principal Ambrosio O’Higgins, quien realizó el parlamento más costoso de la época: el de Negrete en 1793.

En medio del conflicto, el que no se debe entender como un enfrentamiento continuado sino más bien intermitente, se desarrolló el Parlamento de Negrete en 1771. En este se hicieron múltiples referencias a la extensa frontera al sur de la línea del Bío-Bío, descartándose la acepción a variados espacios fronterizos, entendiéndose que correspondía a la suma de los territorios bajo control de los butalmapus "o Parcialidades de todos los Yndios que avitan desde el famoso Rio de Biobio hasta las inmediaciones de Valdivia, y de mar a Cordillera incluso los Pehuenches"56, refiriéndose al vínculo frontera-butalmapus, como: "se ha lebantado a fin de Celebrar en ella Parlamento con los quatro Butalmapus (...) en que se establecen solidas Pazes con el maior decoro de nuestras armas, y la maior seguridad, y sociego de toda esta frontera" ${ }^{57}$, presentándose la frontera como una unidad territorial extensa, es decir, como una macrorregión que iba desde la línea del Bío-Bío al sur.

Una década más tarde, para el parlamento celebrado en Santiago en 1782, se mantuvo la visión hispana de un espacio macrorregional fronterizo. En esta junta general es interesante la referencia, aunque en menor proporción, a los butalmapus como cuerpos territoriales que poseían frontera propia. En este sentido, se manifestó: "Muy Ylustre Señor Presidente = A consequencia de lo que llevo participado à V. S. antes de mi salida de la Concepcion para estas Fronteras sobre el enbio de los quatro Casiques uno de cada Butalmapu"58. Para el parlamento celebrado dos años más tarde, en Lonquilmo en 1784, continuaron las referencias delimitatorias sobre dichos cuerpos territoriales independientes a la macrorregión-frontera. Lo curioso de esta junta general es que continuaron las menciones a cuerpos territoriales con dichas delimitaciones (aunque en menor proporción), incluyendo el territorio conocido como Puelmapu (actual territorio argentino). Aquí, se estableció que serán "en adelante tambien comprehendidos en este mismo Butalmapu los Puelches y Yndios Pampas que poseen los Paises a la parte septentrional del Reyno desde Malalgue y Fronteras de Mendoza, hasta el Mamilmapu situado en las Pampas de Buenos Ayres"59.

En contraste, a fines del siglo XVIII, en el parlamento llevado a cabo en Negrete en 1793, se hizo alusión a los cuatro butalmapus, los que formaban la frontera. Se señaló: "No pudiendo el

\footnotetext{
${ }^{56}$ Parlamento de Negrete de 1771, BNCh, Ms.M, t. 332, fs. 518-566; APUD Zavala. 2015. Los Parlamentos HispanoMapuches..., p. 287.

${ }^{57}$ Parlamento de Negrete de 1771, BNCh, Ms.M, t. 332, fs. 518-566; APUD Zavala. 2015. Los Parlamentos HispanoMapuches..., p. 287.

58 Parlamento de Santiago de 1782, AGI, Chile, 224, fs. 1-20; APUD Zavala. 2015. Los Parlamentos HispanoMapuches..., p. 335.

59 Parlamento de Lonquilmo de 1784, AGI, Chile, 193, fs. 1-32v; APUD Zavala. 2015. Los Parlamentos HispanoMapuches..., p. 354.
} 
Ilustrisimo Señor Obispo Don Francisco Josef Morán asistir personalmente al Parlamento general con los Indios de los quatro Butalmapus de esta frontera"60. Diez años más tarde, a inicios del siglo XIX, y como parte de los últimos parlamentos celebrados entre hispanos y mapuches, en Negrete en 1803 se repitió la asociación anterior con los cuatro Butalmapus, señalándose, además, la existencia de "Governadores" y "Capitanejos" mapuches, los que eran "los Governadores de los quatro Butalmapus con doscientos treinta y nueve Caciques, y cantidad de Capitanejos y respetados hasta el numero de mil y tantas personas"61. En estas últimas juntas generales estudiadas se deja de manifiesto el imaginario hispano de un espacio macrorregional mapuche vinculada a los butalmapus. Es más, si se estudian las referencias parlamentarias entre fines del siglo XVIII e inicios del XIX, la idea de la frontera como cuerpo territorial mapuche es clara, a pesar de una subdivisión interna asociada el butalmapu. En suma, con respecto al papel de los butalmapus, estos dan cuenta de la importancia que estaban adquiriendo estas conformaciones territoriales al ser percibidos como cuerpos territoriales que constituían la frontera. Es interesante la teoría de Eduardo Téllez, quien establece que desde el Estado Hispánico existió la intención de unificar políticamente por medio de la creación del butalmapu al multicéfalo territorio mapuche, lo que finalmente no sucedió por la lógica segmentaria de dicha sociedad ${ }^{62}$.

En el cuadro 1 se presenta la frecuencia en el empleo del concepto "fronteras" y "frontera" en los parlamentos hispano-mapuches, entre 1605 y 1803, con el que se podrá tener una visión general de larga duración, la que permite cuantificar las referencias y brinda una visión de conjunto acerca de la percepción hispana del territorio.

\footnotetext{
${ }^{60}$ Parlamento de Negrete de 1793, AGS, SGU, 6894, exp. 11, fs. 1-43; APUD Zavala. 2015. Los Parlamentos HispanoMapuches..., p. 382.

61 Parlamento de Negrete de 1803, AGI, Chile, 204, fs. 1-17; APUD Zavala. 2015. Los Parlamentos HispanoMapuches..., p. 413.

62 Téllez L., Eduardo. 2009. De la Behetría al Vutamapu: una exploración en torno al potencial de integración política del pueblo mapuche. Araucanía, 1545-1810, tesis de doctorado, Universidad de Chile, Santiago, Chile, pp. 227-228.
} 
Cuadro $\mathrm{N}^{\circ}$ 1: trayectoria histórica de las referencias hispanas al espacio situado al sur del Bío-Bío

\begin{tabular}{|c|c|c|c|}
\hline \multirow{2}{*}{ Lugar } & \multirow{2}{*}{ Fecha } & \multicolumn{2}{|c|}{ Cuantificación de las referencias, como } \\
\hline & & "Fronteras" & "Frontera" \\
\hline Concepción & 1605 & 1 & 1 \\
\hline Catiray & 1612 & - & - \\
\hline Arauco & 1612 & - & - \\
\hline Paicaví & 1612 & - & - \\
\hline Nacimiento & 1617 & 6 & 2 \\
\hline Quillín & 1641 & 1 & - \\
\hline Quillín & 1647 & 2 & - \\
\hline Santa María de Guadalupe & 1662 & 2 & 3 \\
\hline San Felipe de Austria & 1663 & 1 & 1 \\
\hline Concepción & 1665 & - & 1 \\
\hline Malloco & 1671 & 1 & - \\
\hline Yumbel & 1692 & - & 1 \\
\hline Choque-Choque & 1694 & 1 & 2 \\
\hline Purén & 1698 & 3 & 2 \\
\hline Tapihue & 1716 & - & 8 \\
\hline Negrete & 1726 & - & 1 \\
\hline Tapihue & 1738 & - & - \\
\hline Tapihue & 1746 & - & 1 \\
\hline Concepción & 1759 & - & 4 \\
\hline Santiago & 1760 & 1 & 6 \\
\hline Nacimiento & 1764 & - & 1 \\
\hline Negrete & 1771 & - & 14 \\
\hline Santiago & 1772 & - & 7 \\
\hline Tapihue & 1774 & - & 3 \\
\hline Santiago & 1782 & 1 & 18 \\
\hline Lonquilmo & 1784 & 7 & 17 \\
\hline Negrete & 1793 & 1 & 30 \\
\hline Negrete & 1803 & - & 5 \\
\hline
\end{tabular}

Fuente: elaboración propia en base a: Zavala, José, Los Parlamentos Hispano-Mapuches, 1593-1803: Textos Fundamentales, 2015.

Del cuadro se puede deducir que, durante la primera mitad del siglo XVII, entre 1605 y 1647, es mayoritario el empleo de la conceptualización múltiple, es decir, "fronteras". En cambio, entre 1662 y 1698 existió una cierta equivalencia en el empleo de los conceptos "fronteras" y 
"frontera". La "frontera" como una amplia macro región se hizo manifiesta en el siglo XVIII, desde 1716 y lo que restó del período colonial. Es interesante que esto tiene correlación con lo que plantea Venegas para la larga duración, entre los siglos XVI y XVIII. Para las dos primeras centurias, establece que no es correcto referirse a la población originaria desde una perspectiva integradora, es decir, como "Mapuche", al pensar que se trataba de sociedades segmentadas ${ }^{63}$. Para el XVIII se hace partícipe de la teoría de Boccara, "en el sentido que el Pueblo Mapuche como lo conocemos en el presente surge por un proceso de etnogénesis hacia el siglo XVIII"164, la que se explicaría por las constantes relaciones con los españoles. Es decir, que la percepción hispana allende el Bío-Bío del espacio fronterizo estuvo relacionada con la integración sociopolítica mapuche en un período de larga duración.

\section{Conclusiones}

Al realizar el estudio parlamentario de larga duración, el que conllevó la revisión de los tratados diplomáticos hispano-mapuches entre 1605 y 1803, se pudo observar un escenario difuso en cuanto al carácter histórico uniforme del control territorial de la zona fronteriza al sur del BíoBío.

Con respecto a esto, durante la primera mitad del siglo XVII (1605-1647) se vibiliza, desde el lenguaje hispano, constantes referencias a las fronteras al sur, es decir, allende al Bío-Bío o "la raya". Las referencias a la existencia de espacios territoriales mapuches con fronteras propias, habla, desde la percepción hispana, acerca de la multiplicidad de pueblos o agrupaciones al sur.

Durante la segunda mitad del siglo XVII (1662-1698) la división territorial o la percepción hispana de diversos pueblos allende al sur comienza a contrastar con la idea de un espacio uniforme o de frontera. Desde una perspectiva de la frecuencia en el uso del término, hay una equivalencia, lo que podría estar vinculado a la concepción hispana de un espacio culturalmente cohesionado, al reconfigurarse el espacio como uno donde primarían las interrelaciones socioeconómicas antes que las bélicas.

Durante el siglo XVIII e inicios del XIX (1716-1803) la percepción hispana del territorio allende al Bío-Bío tuvo decididamente relación con la idea de un espacio fronterizo uniforme. Esta tendencia vinculada a percibir la existencia de una macro región mapuche constituida de múltiples agrupaciones locales existió hasta fines del siglo XVIII. En parte de lo que restó del período colonial se visibilizó la idea del butalmapu con su frontera independiente, mostrándolo como un espacio escindido; aunque, en mayor frecuencia, en el mismo período también se mostró a los butalmapus como los cuerpos que formaban la frontera.

\footnotetext{
${ }^{63}$ Venegas, F. 2014. De Tralca-Mawida a..., p. 27.

64 Ídem.
} 
En suma, se visibiliza una trayectoria hacia la integración territorial al sur del Bío-Bío desde la óptica que ofrecen los parlamentos escritos, entendidos estos como encuentros diplomáticos desde la percepción hispana del momento. Estos hacen posible establecer que existieron tres períodos para su conformación; el primero, 1605-1647, caracterizado por la disgregación fronteriza allende al Bío-Bío; el segundo, 1662-1698, por el carácter ambivalente o de indefinición en cuanto a la conformación; y el tercero, 1716-1803, por la percepción de una macro región de frontera, la que se ubicaba al sur del Bío-Bío hasta la altura del territorio valdiviano (en sentido norte-sur) y desde el mar hasta la Cordillera de los Andes (en sentido este-oeste), aunque en contadas ocasiones se incluyera al Puelmapu allende la Cordillera en el actual territorio argentino.

\section{Referencias bibliográficas}

Alonso, I. y Payás, G. 2009. "La mediación lingüística institucionalizada en las fronteras Hispano-mapuche e Hispano-árabe: ¿un patrón similar?", en Historia № 42, Santiago, pp. 185-201.

Barros, D. 1999. Historia General de Chile, Tomo III, Santiago, Editorial Universitaria.

Barros, D. 1999. Historia General de Chile, Tomo IV, Santiago, Editorial Universitaria.

Bengoa, J. 2000. Historia del pueblo mapuche. Siglos XIX y XX, Santiago, LOM.

Bengoa, J. 2003. Historia de los antiguos mapuches del sur, Santiago, Catalonia.

Bengoa, J. 2007. El tratado de Quilín, Santiago, Catalonia.

Braudel, F. 2006. "La larga duración", en Revista Académica de Relaciones Internacionales №5, Santiago, pp. 1-36.

Carreño, L. 2009. "Relaciones fronterizas y violencia en la plaza fuerte de Valdivia. Siglo XVIII", en Espacio Regional $\mathrm{N}^{\circ}$ 6, Osorno, pp. 13-22.

Carvallo, V. 1875. Descripción históricio-geográfica del Reino de Chile, Santiago, Imprenta del Ferrocarril. Cerda-Hegerl, P. 1997. Fronteras del Sur. La Región del Bío-Bío y la Araucanía chilena, 1604-1883, Temuco, Ediciones Universidad de la Frontera.

Curivil, R., Payás, G. y Zavala, J. 2014. "La palabra "Parlamento" y su equivalente en mapudungún en los ámbitos colonial y republicano. Un estudio sobre fuentes chilenas bilingües y de traducción", en Historia, $N^{\circ} 47$, Santiago, pp. 355-373.

Dillehay, T. y Zavala, J. 2010. "El "Estado de Arauco" frente a la conquista española: estructuración sociopolítica y ritual de los araucano-mapuches en los valles nahuelbutanos durante los siglos XVI y XVII", en Chungará N² 2, Arica, pp. 433-450.

Dillehay, T., Payás, G. y Zavala, J. (2013). "El requerimiento de Martín García Óñez de Loyola a los indios de Quilacoya, Rere, Taruchina y Maquegua de 1593, testimonio oficial de Parlamentos Hispanomapuches tempranos", en Memoria Americana N²1, Buenos Aires, pp. 235-268.

Dillehay, T., Le Bonniec, F., Payás, G., y Zavala, J. 2015. "Los parlamentos hispano-mapuches como espacios de reconocimiento del Otro enemigo: huellas históricas, lingüísticas y territoriales", en Araguás, I., Páez, A., y Samaniego, M. (eds.). Traducción y representaciones del conflicto desde España y América. Una perspectiva interdisciplinar, Salamanca, Ediciones Universidad de Salamanca, pp. 3548.

Foerster, R. y Vergara, J. 1993. "¿'Relaciones interétnicas o relaciones fronterizas?", en Revista de Historia Indígena $\mathrm{N}^{\circ} 1$, Santiago, pp. 9-33. 
Góngora, M. 1966. Vagabundaje y sociedad fronteriza en Chile, siglos XVII a XIX, Santiago, Universidad de Chile.

Illanes, M. 2014. "La cuarta frontera valdiviana. El caso del territorio valdiviano (Chile, XVII-XIX)", en Atenea $\mathrm{N}^{\circ}$ 509, Concepción, pp. 227-243.

Leiva, A. 1985. El primer avance de la Araucanía: Angol 1862. Temuco, Ediciones Universidad de la Frontera.

León, L. 1990. Maloqueros y Conchavadores en Araucanía y las Pampas, 1700-1800, Temuco, Ediciones Universidad de la Frontera.

León, L. 1993. El Parlamento de Tapihue, 1774, Santiago, Ediciones Rehue.

Méndez, L. 2014. "Diálogo entre españoles e indígenas en los Parlamentos de los siglos XVI y XVII", en Cornejo, A. (ed.). Coyunturas pasadas y presentes de los pueblos originarios, Santiago, Catalonia, pp. 13-81.

Payás, G. y Zavala, J. 2015. "Ambrosio O’Higgins y los Parlamentos Hispano-mapuches, 1771-1803: política indígena, escritura administrativa y mediación lingüístico-cultural en la época borbónica chilena", en Memoria Americana N²3, Buenos Aires, pp. 103-136.

Pinto, J. 1988. "Frontera, misiones y misioneros en Chile. La Araucanía, 1600-1900", en Casanova, H., Matthei, M., Pinto, J. y Uribe, S. Misioneros en la Araucanía, Temuco, Ediciones Universidad de la Frontera.

Pinto, J. 1996. Araucanía y Pampas. Un mundo fronterizo en América del Sur, Temuco, Ediciones Universidad de la Frontera.

Reitano, E. y Truchuelo, S. 2017. "A propósito de Las fronteras en el mundo atlántico (siglos XVI-XIX)", en Reitano, E. y Truchuelo, S. (eds.). Las fronteras en el Mundo Atlántico (siglos XVI-XIX), Mar del Plata, Universidad Nacional de La Plata, pp. 17-26.

Ruiz-Esquide, A. 1993. Los indios amigos en la frontera araucana, Santiago, DIBAM.

Téllez, E. 2009. De la Behetría al Vutamapu: una exploración en torno al potencial de integración política del pueblo mapuche. Araucanía, 1545-1810, Santiago, Chile, Universidad de Chile, tesis para optar al grado de doctor.

Urbina, M. 2009. La Frontera de Arriba en Chile Colonial. Interacción hispano-indígena en el territorio entre Valdivia y Chiloé e imaginario de sus bordes geográficos, 1600-1800, Valparaíso, Ediciones Universidad de Valparaíso.

Venegas, F. 2014. De Tralca-Mawida a Santa Juana. Despliegue Histórico de una Localidad en la Frontera del Biobío, Valparaíso, Ediciones Universitarias de Valparaíso.

Villalobos, S. 1995. Vida fronteriza en Chile: el mito de la Guerra de Arauco, Santiago, Andrés Bello.

Villalobos, S. 2003. Chile y su historia, Santiago, Editorial Universitaria.

Zavala, J. 2005. "Aproximación antropológica a los parlamentos hispano-mapuches del siglo XVIII", en Revista Austerra $\mathrm{N}^{\circ} 2$, Santiago, pp. 49-58.

Zavala, J (ed.). 2015. Los Parlamentos Hispano-Mapuches, 1593-1803: Textos Fundamentales, Temuco, Ediciones Universidad Católica de Temuco. 